\title{
Influencia de la transformación austenita-martensita en la estabilidad dimensional de un nuevo acero para herramientas aleado con niobio $(0,08 \%)$ y vanadio $(0,12 \%)$
}

\author{
Gerardo Conejero Ortega ${ }^{\mathrm{a}, \bowtie}$, Nuria Candela Vázquez ${ }^{\mathrm{a}}$, Montserrat Pichel Martínez ${ }^{\mathrm{a}}$, \\ Rafael Barea Del Cerro ${ }^{a}$, Manuel Carsí Cebrián ${ }^{b}$ \\ ${ }^{a}$ Escuela Politécnica Superior (EPS) Universidad Nebrija, c/ Pirineos, 55, 28040 Madrid, España \\ ${ }^{\mathrm{b}}$ Centro Nacional de Investigaciones Metalúrgicas (CENIM).CSIC. Avda. Gregorio del Amo, 8, 28040 Madrid, España \\ Autor para la correspondencia: gconejer@nebrija.es
}

Enviado: 14 Junio 2013; Aceptado: 16 Octubre 2013; Publicado on-line: 26 August 2014

\begin{abstract}
RESUMEN: Se ha estudiado la influencia de la transformación de austenita a martensita en la estabilidad dimensional de un acero experimental para herramientas aleado con niobio $(0,08 \%$ en peso) y vanadio $(0,12 \%$ en peso). La estabilidad dimensional del acero experimental fue comparada con la de un acero comercial antes y después de ser sometidos, ambos aceros, a dos tratamientos térmicos diferentes. Los tratamientos térmicos consistieron en un calentamiento y mantenimiento durante una hora a temperaturas de $860{ }^{\circ} \mathrm{C}(\mathrm{T} 1)$ y $900{ }^{\circ} \mathrm{C}(\mathrm{T} 2)$ y un enfriamiento final en horno con nitrógeno $\left(\mathrm{N}_{2}\right)$ a 1 atmósfera de presión. Inicialmente, se determinaron las composiciones del acero experimental y comercial, mediante técnica de luminiscencia (GDL), y las temperaturas de transformación $\mathrm{Ac}_{1}$ y $\mathrm{Ac}_{3}$ del acero experimental, mediante ensayos dilatométricos, para seleccionar las temperaturas de austenización de los tratamientos T1 y T2. Posteriormente, tras medir la dureza de ambos aceros, se caracterizó la microestructura de los dos aceros mediante técnicas de Difracción de Rayos X (XRD) y metalografía óptica, antes y después de los tratamientos térmicos T1 y T2. Por último, se realizaron los análisis de estabilidad dimensional longitudinal y angular por medio de las medidas de las probetas anulares de los aceros comercial y experimental. Los resultados obtenidos tras haber realizado un contraste de hipótesis mostraron que la variación relativa longitudinal máxima calculada para el acero experimental fue del $0,2 \%$ y su variación relativa angular no fue significativa.
\end{abstract}

PALABRAS CLAVE: Aceros de herramientas; Austenita; Estabilidad dimensional; Martensita; Niobio; Transformaciones de fase; Vanadio

Citation / Cómo citar este artículo: Conejero G., Candela, N., Pichel, M., Barea Del Cerro, R., Carsí, M. (2014) "Influencia de la transformación austenita-martensita en la estabilidad dimensional de un nuevo acero para herramientas aleado con niobio (0,08\%) y vanadio (0,12\%)". Rev. Metal. 50(3): e018. doi: http://dx.doi.org/10.3989/ revmetalm.018.

ABSTRACT: Influence of the austenite-martensite transformation in the dimensional stability of a new tool steel alloyed with niobium $(0.08 \% \mathrm{wt}$.) and vanadium $(0.12 \% \mathrm{wt}$. $)$. Austenite-martensite transformation influence on the dimensional stability of a new experimental tool steel alloyed with niobium $(0.08 \% \mathrm{wt}$.) and vanadium $(0.12 \%$ wt. $)$ has been studied. The dimensional stability of this new steel was compared with the dimensional stability of commercial steel, after and before two thermal treatments, $\mathrm{T} 1\left(860^{\circ} \mathrm{C}\right)$ and $\mathrm{T} 2\left(900^{\circ} \mathrm{C}\right)$. The thermal treatments consisted on heating and cooling, at 1 atmosphere of pressure, in $\mathrm{N}_{2}$ atmosphere furnace, following by heating in a conventional furnace at $180{ }^{\circ} \mathrm{C}$ during 1 hour. Initially, the experimental steel composition and $\mathrm{Ac}_{1}$ and $\mathrm{Ac}_{3}$ transformation temperatures were determined by glow-discharge luminescence (GDL) and 
dilatometric tests, respectively, in order to select the austenization temperatures of T1 and T2 treatments. After hardness measurement, the microstructure of both steels was characterized by X-Ray Diffraction (XRD) and optical metallography, before and after of T1 and T2 thermal treatments. Finally, longitudinal and angular dimensional stability analyses were realized for both commercial and experimental steels. After a contrastive hypothesis analysis, the results showed that the longitudinal relative variation of the experimental steel calculated was around $0.2 \%$ and the angular relative variation was not significant.

KEYWORDS: Austenite; Dimensional stability; Martensite; Niobium; Phase transformations; Tool steel; Vanadium

Copyright: (C) 2014 CSIC. This is an open-access article distributed under the terms of the Creative Commons Attribution-Non Commercial (by-nc) Spain 3.0 License.

\section{INTRODUCCIÓN}

Los materiales que apenas varían sus dimensiones originales tras ser sometidos a procesos termomecánicos o fuerzas externas durante los procesos de elaboración y de funcionamiento en servicio son estables dimensionalmente. Por el contrario, los materiales que son inestables dimensionalmente tienden a modificar sus dimensiones debido a la acción de tensiones externas, tensiones internas, la anisotropía, y los cambios microestructurales en el material (Paquin, 1990).

La distorsión producida en un acero cuando es sometido a un proceso termo-mecánico se origina por la presencia de varios factores de naturaleza térmica, mecánica y microestructural que influyen conjuntamente e interaccionan de manera simultánea (Canale y Totten, 2005; Arimoto et al., 2009; Nallathambi et al., 2010). En la mecánica del continuo la distorsión se analiza por medio del campo de deformaciones del material. La deformación puede ser debida a solicitaciones mecánicas (elástica y plástica) y también de origen térmico, causada por gradientes térmicos, transformaciones de fase en el interior del material y por fluencia plástica ("creep"). La suma de cada tipo de deformación origina una deformación total $\left(\varepsilon_{i, j}{ }^{\mathrm{T}}\right)$ en cada punto del material e instante de tiempo que es la suma de los diferentes tipos de deformación y que puede expresarse mediante la ecuación (1):

$$
\varepsilon_{i, j}{ }^{\mathrm{T}}=\varepsilon_{\mathrm{i}, \mathrm{j}}{ }^{\mathrm{E}}+\varepsilon_{\mathrm{i}, \mathrm{j}}{ }^{\mathrm{P}}+\varepsilon_{\mathrm{i}, \mathrm{j}}{ }^{\mathrm{TH}}+\varepsilon_{\mathrm{i}, \mathrm{j}}{ }^{\mathrm{TR}}+\varepsilon_{\mathrm{i}, \mathrm{j}}{ }^{\mathrm{C}}
$$

Las deformaciones corresponden a: $\varepsilon_{\mathrm{i}, \mathrm{j}}^{\mathrm{E}}$, deformación elástica de origen mecánico; $\varepsilon_{\mathrm{i}, \mathrm{j}}{ }^{\mathrm{P}}$, deformación plástica de origen mecánico; $\varepsilon_{\mathrm{i}, \mathrm{j}}{ }^{\mathrm{T}}$, deformación térmica ocasionada por gradientes térmicos; $\varepsilon_{\mathrm{i}, \mathrm{j}}^{\mathrm{TR}}$, deformación originada por transformaciones de fase en el material, debida al aumento (o disminución) de volumen como consecuencia de la variación en los parámetros cristalográficos de las fases originadas respecto de las iniciales y $\varepsilon_{i, j}{ }^{\mathrm{C}}$, deformación por fluencia en caliente debido a las tensiones y temperaturas altas del material.

Todas las deformaciones descritas se originan de manera simultánea durante un proceso termo-mecánico, en mayor o menor medida. Unas deformaciones tendrán más peso específico que otras, dependiendo de la naturaleza y condiciones del proceso. En ausencia de tensiones externas, las deformaciones más importantes se originan por transformación y por gradientes térmicos. Las deformaciones que tienen lugar en un acero como consecuencia de las transformaciones de fase originadas durante un enfriamiento y en ausencia de carga mecánica aplicada son $\varepsilon_{\mathrm{i}, \mathrm{j}}^{\mathrm{TH}}$ y $\varepsilon_{\mathrm{i}, \mathrm{j}}^{\mathrm{TR}}$.

La expresión para $\varepsilon_{i, j}{ }^{\mathrm{TH}}$ a una temperatura $\mathrm{T}$ para una determinada dirección de deformación viene definida por la fracción de fase $\left(\mathrm{X}_{\mathrm{A}, \mathrm{M}}\right)$ y el coeficiente de dilatación térmica $\left(\alpha_{\mathrm{A}, \mathrm{M}}\right)$ para la austenita retenida y la martensita, respectivamente y puede expresarse mediante la ecuación (2):

$$
\varepsilon_{i, j}{ }^{T H}=\left(X_{A} \alpha_{A}+X_{M} \alpha_{M}\right)_{i, j} \cdot T
$$

Esta expresión muestra cómo la deformación por tensión térmica está relacionada con la proporción de fases del acero. El valor del coeficiente de la austenita es mayor que el de la martensita (Bhadhesia, 2002; Qiu et al., 2009) por lo que, durante la transformación, la compresión de la austenita es mayor que la de la martensita, induciéndose tensiones internas como consecuencia del gradiente de deformación.

La deformación $\varepsilon_{\mathrm{i}, \mathrm{j}}{ }^{\mathrm{TR}}$ es característica de la transformación austenita-martensita y es debida, principalmente, a la diferencia entre el parámetro de red de la estructura cristalina de la martensita y el de la austenita y puede ser expresada mediante la ecuación (3):

$$
\varepsilon_{i, j}{ }^{T R}=\left(\beta_{A-M} X_{M}\right)_{i, j}
$$

El parámetro $\beta_{\mathrm{A}-\mathrm{M}}$ describe la dilatación por la transformación de un porcentaje de fase martensítica $\mathrm{X}_{\mathrm{M}}$ (Yao et al., 2004). La transformación austenitamartensita es no-difusional (displaciva) y se caracteriza por el desplazamiento homogéneo de una red de átomos respecto de su posición inicial de equilibrio hasta alcanzar una posición metaestable termodinámicamente. La transformación empieza y termina a unas temperaturas denominadas $\mathrm{M}_{\mathrm{S}} \mathrm{y} \mathrm{M}_{\mathrm{F}}$, respectivamente. Por otra parte, la temperatura $\mathrm{M}_{\mathrm{S}}$ desciende cuando aumenta el contenido de carbono en el acero (Nallathambi et al., 2010) y por lo tanto, 
los aceros con mayor contenido en carbono tienden a retener más cantidad de austenita. Para calcular el valor de la temperatura $\mathrm{M}_{\mathrm{S}}$ se utilizan ecuaciones fenomenológicas, como la descrita por Steven y Haynes, (1956), válida para aceros que contengan hasta un $0,5 \%$ de carbono en peso $\left(\mathrm{x}_{\mathrm{C}}=0,5\right)($ Ec. 4$)$. El resultado obtenido depende también de las composiciones, en tanto por ciento en peso, de otros elementos de aleación presentes en el acero.

$$
\begin{aligned}
\mathrm{M}_{\mathrm{S} \text { (Steven-Haynes) }} & =561-474 \mathrm{x}_{\mathrm{C}}-33 \mathrm{x}_{\mathrm{Mn}} \\
& -17 \mathrm{x}_{\mathrm{Ni}}-17 \mathrm{x}_{\mathrm{Cr}}-21 \mathrm{x}_{\mathrm{Mo}}
\end{aligned}
$$

Resulta interesante analizar cómo se produce la deformación denominada $\varepsilon_{\mathrm{i}, \mathrm{j}}^{\mathrm{TR}}$ en la transformación austenita-martensita (Santofimia, 2006). La estructura centrada en las caras (FCC) de la austenita, se transforma en una estructura de no equilibrio tetragonal centrada en el interior (BTC) característica de la martensita. Su estructura tiene una relación de ejes cristalográficos de $\mathrm{a}=\mathrm{b} \neq \mathrm{c}$, donde la relación c/a es una medida de la tetragonalidad de la martensita cuyo valor depende del contenido de carbono. En la transformación austenita-martensita se observa un aumento en volumen provocado por una contracción del $20 \%$ en el eje c y una dilatación del $12 \%$ a lo largo de los ejes a y b, denominada deformación de Bain (Santofimia, 2006). Por último, la transformación martensítica lleva asociada otra distorsión provocada por el deslizamiento de dislocaciones. La acción conjunta y simultánea de ambas deformaciones da lugar a la deformación de plano invariante (IPS) con componente de cizalladura, caracterizada por generar, de manera simultánea, una dilatación uniaxial y una deformación angular en una dirección perpendicular a la anterior.

De igual forma, existen dos factores que pueden afectar a la estabilidad dimensional de la pieza enfriada. Por una parte, la fase que se denomina austenita retenida permanece en el acero después del enfriamiento y depende de las temperaturas $\mathrm{M}_{\mathrm{S}}$ y $\mathrm{M}_{\mathrm{F}}$ (Canale y Totten, 2005). El porcentaje de austenita retenida será mayor cuanto más bajas sean las temperaturas $\mathrm{M}_{\mathrm{S}}$ y $\mathrm{M}_{\mathrm{F}}$ para un determinado acero, ya que si la temperatura final de enfriamiento es superior a la temperatura $\mathrm{M}_{\mathrm{F}}$, la transformación austenita-martensita es incompleta. La cantidad de austenita retenida obtenida durante un enfriamiento continuo ha sido estudiada desde hace décadas (Yao et al., 2004; Telher, 2009; Nallathambi et al., 2010) y se ha demostrado que afecta a la inestabilidad dimensional en procesos posteriores de revenido, y su valor da una idea inicial de la capacidad de transformación de la austenita en el acero después de haber realizado un determinado tratamiento térmico. Por otra parte, la tendencia al agrietamiento de un acero durante un enfriamiento está relacionada con el contenido en carbono equivalente $\left(\mathrm{C}_{\mathrm{eq}}\right)$ (Canale y Totten, 2005) que depende de su composición química en porcentaje en peso. El contenido en carbono equivalente viene dado por la expresión (5):

$$
\begin{aligned}
\mathrm{C}_{\mathrm{eq}} & =\mathrm{x}_{\mathrm{C}}+\left(\mathrm{x}_{\mathrm{Mn}}\right) / 5+\left(\mathrm{x}_{\mathrm{Cr}}\right) / 4+\left(\mathrm{x}_{\mathrm{Mo}}\right) / 3+\left(\mathrm{x}_{\mathrm{Ni}}\right) / 10 \\
& +\left(\mathrm{x}_{\mathrm{V}}\right) / 5+\left(\mathrm{x}_{\mathrm{Si}}-0,5\right) / 5+\left(\mathrm{x}_{\mathrm{Ti}}\right) / 5+\mathrm{x}_{\mathrm{W}} / 10+\mathrm{x}_{\mathrm{Al}} / 10
\end{aligned}
$$

El agrietamiento se origina debido a la concentración de esfuerzos por la presencia de defectos tales como agujeros, bordes en ángulo recto y otras heterogeneidades geométricas, que se ve favorecida por la presencia de defectos microscópicos en el material como segregación de carburos y nitruros.

La influencia de los elementos de aleación sobre la transformación austenita-martensita está relacionada con el porcentaje de carbono en la composición del acero, como se ha comentado anteriormente. El acero experimental tiene presencia de niobio y vanadio $(0,08$ y $0,12 \%$ en peso, respectivamente) en su composición. Estos elementos de aleación presentan una alta afinidad para formar carburos, y un producto de solubilidad elevado. Esto significa que los carburos precipitan de manera muy dispersa durante la austenización y actúan de manera muy eficiente sobre el control del tamaño de grano austenítico (Fossaert et al., 1995; San Martín et al., 2006; Yang y Bhadhesia, 2009). Los carburos actúan como elementos inhibidores del crecimiento del tamaño de grano (Gladman, 1966), originando granos finos durante la austenización y aumentando, por lo tanto, la dureza del acero. De esta forma, se consigue un acero con una dureza aceptable a partir de un enfriamiento suave, por lo que se minimiza las inestabilidades dimensionales originadas por la contracción debida al enfriamiento, y la dilatación característica de la transformación austenita-martensita.

La utilización de vanadio como elemento microaleante en determinados aceros se ha desarrollado desde 1950 (Baker, 2009) y la composición de vanadio disuelto en la matriz no influye en la estabilización del campo austenítico (García-Mateo et al., 2005). También hay estudios que hacen referencia al niobio como elemento influyente en la transformación austenita-martensita (Rees et al., 1995) donde muestra el efecto que tiene el niobio y sus carburos sobre la cinética de transformación austenita-martensita. El análisis del efecto del niobio y vanadio depende de las fracciones de composición de ambos elementos disueltos en la matriz y precipitados en forma de carburos. La presencia de mayor cantidad de niobio disuelto en la matriz de austenita tiende a inhibir la transformación austenita-ferrita o austenita-bainita, disminuyendo la velocidad crítica de enfriamiento, definida como la velocidad mínima de enfriamiento con la que se obtiene únicamente martensita como fase transformada. Por otro lado, un aumento de la cantidad de niobio disuelto en la matriz austenítica, durante 
la austenización, aumenta la cantidad de martensita transformada para velocidades críticas de enfriamiento más bajas (Fossaert et al., 1995). Sin embargo, los carburos de niobio aceleran de manera considerable la transformación austenita-bainita, al comportarse como zonas de alta nucleación, actuando de manera opuesta al niobio disuelto en la matriz. De esta manera, una mayor concentración de carburos de niobio puede mejorar las propiedades de dureza pero perjudicar la inhibición de fases no deseadas como la bainita y, sobre todo, la ferrita durante el enfriamiento. El níquel actúa de estabilizador del campo austenítico, y favorece una disminución de la velocidad crítica de enfriamiento del acero sin que se induzcan transformaciones de tipo difusional, como la austenita-ferrita, o de naturaleza más compleja como la transformación austenita-bainita.

En resumen, en este artículo se van a analizar los efectos de la distorsión en muestras de un acero comercial y otro acero experimental nuevo, tras ser sometidos a dos tratamientos térmicos diferentes. Estos tratamientos térmicos corresponden a la etapa más crítica, desde el punto de vista de la distorsión dimensional, de un tratamiento térmico global que abarca varios tratamientos térmicos, posteriores a los que se van a estudiar, pero que tienen un menor efecto sobre la estabilidad dimensional.

\section{MATERIALES Y MÉTODOS}

\subsection{Materiales y técnicas experimentales aplicadas}

Los materiales utilizados en este estudio fueron un acero comercial con $0,12 \% \mathrm{C}$ y un acero experimental nuevo con una composición de $0,22 \% \mathrm{C}$. Las composiciones de estos aceros, se muestran en la Tabla 1.

El acero experimental fue obtenido a partir de una colada realizada en un horno de atmósfera controlada de 70 kilos de capacidad. Posteriormente, se forjaron los lingotes obtenidos a redondos de una longitud de $600 \mathrm{~mm}$ y un diámetro de $10 \mathrm{~mm}$.

El análisis de la composición del acero experimental se realizó por medio de espectrometría de emisión óptica por descarga luminiscente (GD-OES o Glow Discharge Optical Emission Spectrometry) (Skoog, 2007). La técnica se basa en analizar la emisión óptica característica de cada elemento mediante la excitación de la muestra por medio de una fuente externa, en este caso con una lámpara de descarga luminiscente (GDL o Glow Discharge Lamp).

El difractómetro utilizado en los ensayos de difracción fue modelo Siemens D5000 con anillo central abierto de Euler y barrido en X. Para el análisis de los patrones de difracción obtenidos, se utilizó un programa basado en análisis cuantitativo de multifases (Rietveld, 1969).

También se realizaron ensayos de dureza bajo norma UNE-EN-ISO-6508 (2007) en un durómetro Wilson Rockwell/Hardness Tester series 500, antes y después de los tratamientos térmicos. El estudio metalográfico óptico de las microestructuras obtenidas se realizó con un microscopio óptico Olimpus GX51, siendo las muestras atacadas previamente con Nital al $2 \%$.

Los ensayos dilatométricos se realizaron en un dilatómetro universal de alta resolución Adamel Lhomargy DT1000 que permite la programación computerizada de ciclos térmicos en condiciones isotérmicas y anisotérmicas. En cada ensayo se obtuvieron la curva dilatométrica y su correspondiente curva derivada, y las temperaturas críticas relacionadas con las transformaciones de fase se detectan en las zonas de ambas curvas donde aparecen cambios bruscos de pendiente o picos, respectivamente. Las temperaturas $A c_{1}$ y $A c_{3}$ del acero experimental se calcularon mediante una técnica estadística utilizando las curvas derivadas de dilatometría (Mendenhall y Sincich, 1997; Montgomery y Runger, 2003).

Los tratamientos térmicos de calentamiento y enfriamiento (Fig. 1a) se realizaron en gas nitrógeno $\left(\mathrm{N}_{2}\right)$ a 1 atmósfera de presión. El enfriamiento forzado en horno de atmósfera controlada (Fig. 1b) viene justificado por el hecho de que el acero experimental tiene un carbono equivalente (Ec. 5) igual a 0,943 , lo cual indica una probabilidad alta de agrietamiento durante el enfriamiento (Canale y Totten, 2005). Los enfriamientos de aceros en atmósferas de gases son más suaves que en otros medios como agua o aceite, ya que no generan gradientes térmicos elevados entre la superficie y la zona interior de la probeta y se reduce el riesgo de agrietamiento durante el proceso.

Las probetas utilizadas en los tratamientos térmicos para el estudio de la estabilidad dimensional tenían forma de anillo abierto, en el que se mecanizaron tres agujeros de $2 \mathrm{~mm}$ de diámetro (Fig. 1c).

TABLA 1. Composición química de los aceros comercial y experimental

\begin{tabular}{lccccccccccccc}
\hline & \multicolumn{10}{c}{ Elemento (\% en peso) } \\
\cline { 2 - 12 } Acero & $\mathbf{C}$ & $\mathbf{M n}$ & $\mathbf{S i}$ & $\mathbf{P}$ & $\mathbf{S}$ & $\mathbf{C r}$ & $\mathbf{N i}$ & $\mathbf{M o}$ & $\mathbf{C u}$ & $\mathbf{V}$ & $\mathbf{N b}$ & $\mathbf{F e}$ \\
\hline Comercial & 0,12 & 0,45 & 0,24 & 0,010 & 0,025 & 0,89 & 3,04 & 0,21 & 0,22 & - & - & $\mathrm{B}^{*}$ \\
Experimental & 0,22 & 0,19 & 0,33 & 0,014 & 0,010 & 1,24 & 3,85 & - & 0,15 & 0,12 & 0,08 & $\mathrm{~B}^{*}$ \\
\hline
\end{tabular}

$\mathrm{B}^{*}=$ Balance. 
a)

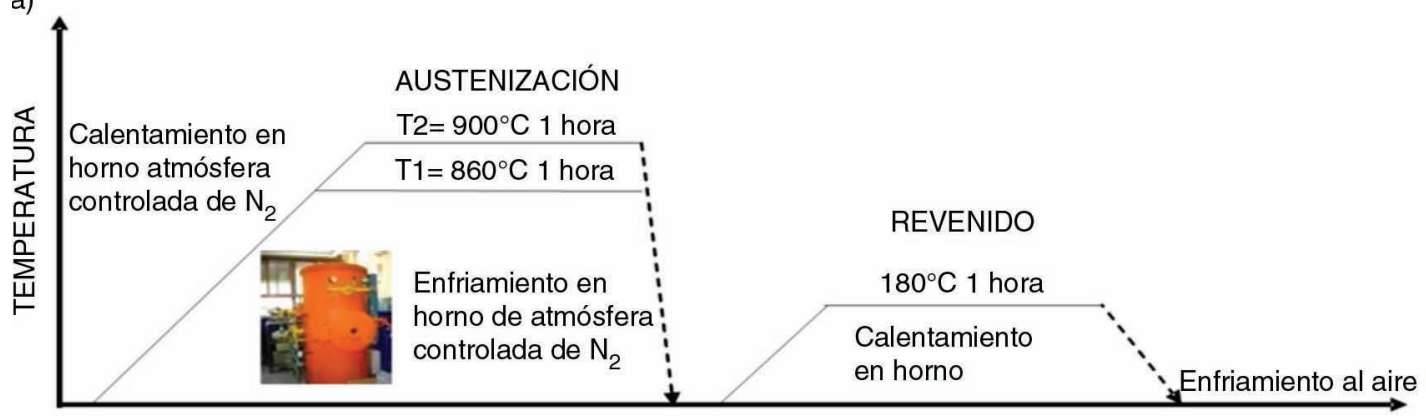

b)
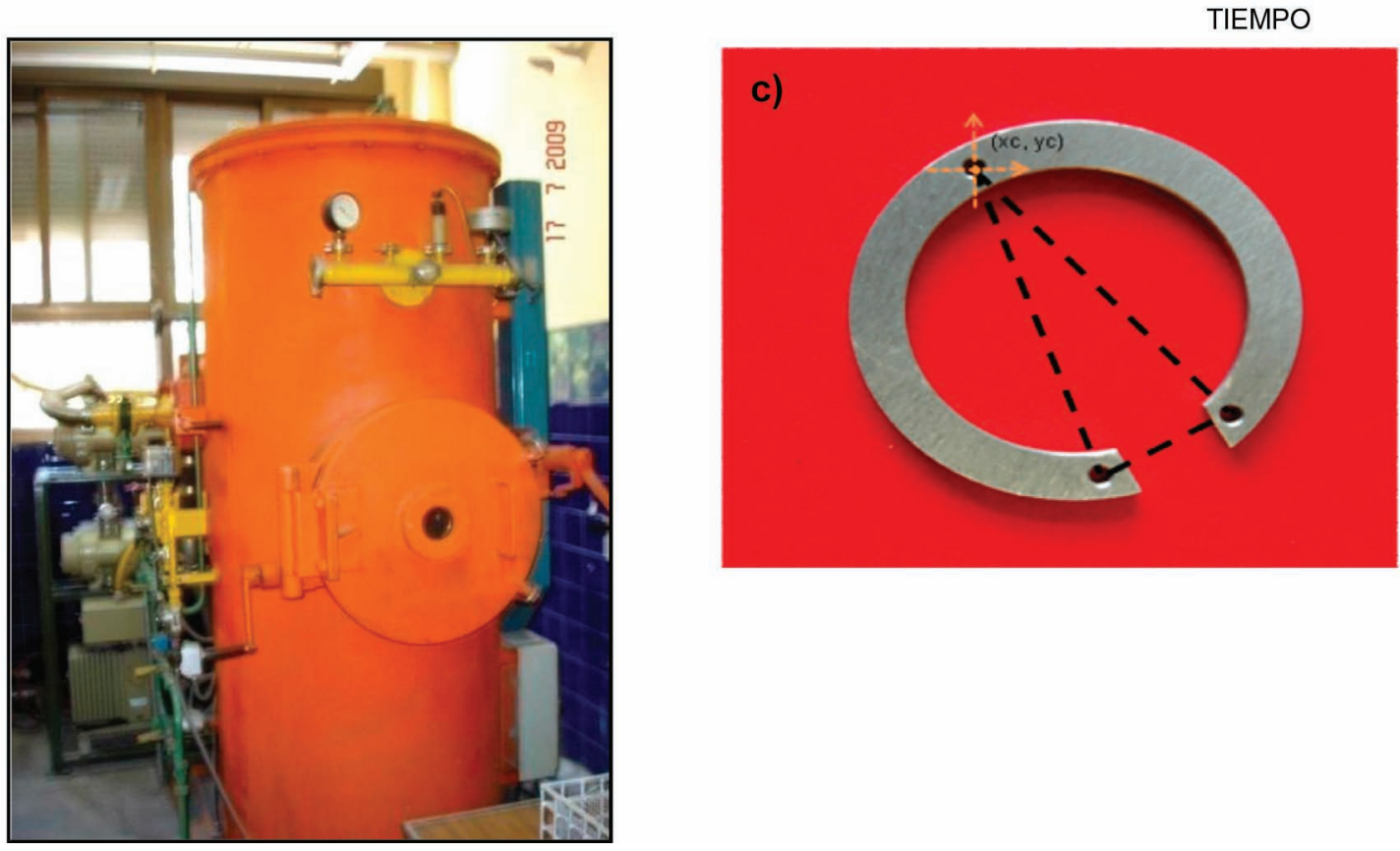

Figura 1. Procesos térmicos T1 y T2 realizados en el horno: a) Diagrama con los ciclos térmicos T1 y T2; b) horno de atmósfera controlada de $\mathrm{N}_{2}$ y pared fría y c) probeta utilizada en los ensayos de estabilidad dimensional donde se muestra el triángulo delimitado por los centros de los agujeros.

En cada agujero se tomaron medidas de las coordenadas de cuatro puntos equidistantes, antes y después de los tratamientos térmicos, mediante un dispositivo formado por un microscopio Nikon MM-1 acoplado a una unidad CPU.

Para comprobar si las diferencias en el valor de las deformaciones obtenidas para el acero comercial y el acero experimental son significativas, se realizó un contraste de hipótesis empleando estadística no paramétrica, mediante la aplicación de la prueba de suma de rangos de Wilcoxon (Mendenhall y Sincich, 1997). Esta prueba no paramétrica compara las distribuciones de probabilidad completas, además de las medianas. En este estudio se aplicó, concretamente, la "prueba de dos extremos" de la suma de rangos de Wilcoxon, donde la hipótesis nula define que las distribuciones de probabilidad asociadas a las dos poblaciones comparadas son equivalentes, mientras que la hipótesis alternativa indica que la distribución de probabilidad de una población está desplazada a la derecha o a la izquierda de la otra. Una vez obtenidos los valores $\mathrm{n}_{1}$, mediciones correspondientes a un grupo y los valores $\mathrm{n}_{2}$, mediciones correspondientes al grupo con el que se compara, el procedimiento de análisis de esta prueba estadística no paramétrica consiste en ordenar desde el menor valor hasta el mayor el número total de muestras $\left(\mathrm{n}_{1}+\mathrm{n}_{2}\right)$ obtenidas. A cada muestra se le asigna un valor numérico (rango) desde el valor 1, que corresponde a la muestra de menor valor. Una vez ordenados y enumerados correspondientemente, se calcula los estadísticos $S_{1}$ y $\mathrm{S}_{2}$, que son la suma de los rangos del grupo $\mathrm{n}_{1} \mathrm{y}$ del grupo $\mathrm{n}_{2}$, respectivamente. Se selecciona la estadística de la prueba, siendo $\mathrm{S}_{1}$ si $\mathrm{n}_{1}<\mathrm{n}_{2}$ o $\mathrm{S}_{2}$ si $\mathrm{n}_{2}<\mathrm{n}_{1}$ (si $\mathrm{n}_{1}=\mathrm{n}_{2}$ se puede usar cualquiera de los dos estadísti$\cos )$. La condición de rechazo de la hipótesis nula se alcanza cuando el estadístico seleccionado $\left(\mathrm{S}_{1}\right.$ o $\left.\mathrm{S}_{2}\right)$ cumpla que $\mathrm{S}_{1,2} \leq \mathrm{S}_{\mathrm{L}}$ o bien $\mathrm{S}_{1,2} \geq \mathrm{S}_{\mathrm{U}}$, donde $\mathrm{S}_{\mathrm{L}}$ y $\mathrm{S}_{\mathrm{U}}$ son dos estadísticos que aparecen en las correspondientes tablas para la prueba de suma de rangos de Wilcoxon. 


\section{PROCEDIMIENTO EXPERIMENTAL}

Inicialmente, se realizaron ensayos de dureza, metalografía y difracción de los aceros en el estado de recepción.

Se llevaron a cabo nueve ensayos dilatométricos a una velocidad de calentamiento de $0,5^{\circ} \mathrm{C} \cdot \mathrm{s}^{-1}$ desde una temperatura ambiente hasta una temperatura de $1000^{\circ} \mathrm{C}$. Posteriormente, se calcularon y analizaron las curvas dilatométricas y sus derivadas en el tramo de calentamiento para cada uno de los nueve ensayos de dilatometría del acero experimental, con el fin de obtener las temperaturas críticas $\mathrm{Ac}_{1}$ y $\mathrm{Ac}_{3}$.

El criterio adoptado fue seleccionar las mismas temperaturas de austenización para los dos aceros y obtener así las mismas condiciones de enfriamiento. Se seleccionaron unas temperaturas de austenización de 860 y $900{ }^{\circ} \mathrm{C}$ para los tratamientos térmicos T1 y T2, respectivamente, que sobrepasan con bastante holgura las temperaturas $\mathrm{Ac}_{3}$ de ambos aceros. Los tratamientos térmicos (Fig. 1a) siguen un ciclo de calentamiento hasta temperaturas de $860{ }^{\circ} \mathrm{C}$ (tratamiento T1) y $900{ }^{\circ} \mathrm{C}$ (tratamiento T2) respectivamente, manteniendo ambas temperaturas durante una hora, y el enfriamiento se realiza con gas $\mathrm{N}_{2}$ a 1 atmósfera de presión. Por último, se realiza un calentamiento de revenido a $180^{\circ} \mathrm{C}$ durante una hora con el objetivo de aliviar tensiones en las muestras después del enfriamiento.

Respecto a los ensayos para calcular la estabilidad dimensional, antes y después de los tratamientos térmicos aplicados, se utilizó una subrutina de la aplicación informática que calculó primero las coordenadas del centro de cada agujero $\left(\mathrm{x}_{\mathrm{c}}, \mathrm{y}_{\mathrm{c}}\right)$ y posteriormente la longitud de los lados y los ángulos originados al unir los centros de cada agujero para cada probeta (Fernández et al., 2002). Posteriormente, se calcularon estadísticamente, para cada tipo de acero y de distorsión, el valor medio, la desviación y error estándar y el rango de valores de variación relativa para un nivel de confianza de 95\% (Mendenhall y Sincich, 1997).

De igual forma, una vez realizados los tratamientos térmicos se realizaron nuevas medidas de dureza, análisis metalográficos y ensayos difractométricos para cada tipo de acero. Por último, se realizó el contraste de hipótesis aplicando estadística no paramétrica.

\section{RESULTADOS Y DISCUSIÓN}

\subsection{Determinación de las temperaturas críticas $A c_{1}$ y $A c_{3}$}

A partir de la información obtenida de las curvas dilatométricas y las correspondientes curvas derivadas, se pueden calcular los valores de la temperatura $\mathrm{Ac}_{1}$ (inicio de la transformación ferrita-austenita durante el calentamiento) y $\mathrm{Ac}_{3}$ (temperatura final de la transformación) ya que, al tratarse de un nuevo acero, ambas temperaturas no están publicadas.

Una vez obtenidas estas temperaturas, se calculó el valor promedio de cada una de ellas obtenido en los nueve ensayos (Fig. 2). Para el acero experimental se obtuvieron unos valores promedio de 726 y $775{ }^{\circ} \mathrm{C}$ para $\mathrm{Ac}_{1}$ y $\mathrm{Ac}_{3}$, respectivamente. Las temperaturas $\mathrm{Ac}_{1}$ y $\mathrm{Ac}_{3}$ del acero comercial se obtuvieron del proveedor y fueron de 690 y $780{ }^{\circ} \mathrm{C}$, respectivamente. Se observa que la temperatura $\mathrm{Ac}_{3}$ del acero experimental es $5{ }^{\circ} \mathrm{C}$ más baja que la del acero comercial. El criterio adoptado fue seleccionar las mismas temperaturas de austenización para los dos aceros y obtener así las mismas condiciones de enfriamiento. Se seleccionaron unas temperaturas de austenización de 860 y $900{ }^{\circ} \mathrm{C}$ para los tratamientos térmicos $\mathrm{T} 1$ y $\mathrm{T} 2$, respectivamente, que sobrepasan con bastante holgura las temperaturas $\mathrm{Ac}_{3}$ de ambos aceros.

\subsection{Análisis difractométrico, de dureza y metalográfico}

Los resultados obtenidos en los ensayos de difracción en el estado de recepción, antes de realizar los tratamientos térmicos, muestran dos patrones de difracción para los aceros comercial y experimental (Figs. 3a y 4a, respectivamente) donde se observan picos de intensidad máximos correspondientes al plano (110) de una estructura cristalina centrada en el cuerpo (BCC). En el patrón de difracción del acero experimental, además, aparecen picos correspondientes a una estructura centrada en las caras (FCC), característica de la $\gamma$-austenita, correspondiendo el pico de máxima intensidad al plano cristalográfico (111). En total, se ha registrado una presencia de FCC $\gamma$-austenita con un porcentaje del 3,7\%.

Los patrones de difracción obtenidos en muestras sometidas a los tratamientos T1 y T2 muestran diferentes valores para el pico (111), correspondiente a la austenita retenida. En el acero comercial, después de ser sometido al tratamiento térmico T1 (Fig. 3b), el análisis determinó que el total de la fase $\gamma$-austenita retenida detectada fue igual a 7,18\%. Por otra parte, después del tratamiento T2 (Fig. 3c) el valor detectado para la $\gamma$-austenita fue de $8,20 \%$. El resto de fase detectada corresponde a una fase BCC característica de la $\alpha$-ferrita.

Para el acero experimental, el diagrama de difracción correspondiente al tratamiento T1 (Fig. 4b) muestra, al igual que en el acero comercial, un pico de intensidad alta correspondiente al plano cristalográfico (110) de una estructura $\alpha$-ferrita (BCC), y un pico de intensidad baja correspondiente al plano (111) que corresponde a una estructura $\gamma$-austenita (FCC). En este caso, el valor total registrado de austenita retenida fue de $6,7 \%$. Por otra parte, para un tratamiento T2 (Fig. 4c), el diagrama muestra también perfiles correspondientes a $\alpha$-ferrita 


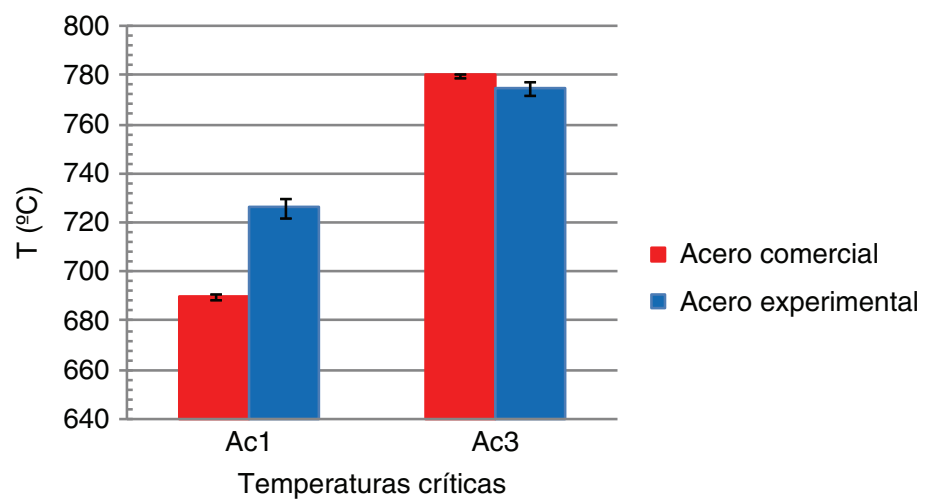

FIgURA 2. Valores promedios de $\mathrm{Ac}_{1}$ y $\mathrm{Ac}_{3}$ para los aceros comercial y experimental.

y $\gamma$-austenita. En este caso, se detectó un valor de austenita retenida de $1,7 \%$, un $75 \%$ menos que la obtenida después del tratamiento T1.

Los diagramas XRD mostrados anteriormente siguen una misma pauta para ambos aceros en todos los tratamientos, consistente en la aparición de un pico máximo de intensidad correspondiente a una estructura $\mathrm{BCC}$ y picos de menor intensidad correspondientes a una estructura FCC (característica de la austenita). Esto evidencia que en ambos aceros ha habido transformación de la austenita, y que la estructura cristalina de la fase resultante tiene estructura BCC, pero no completa el análisis de las muestras, ya que esta información debe ser complementada con los análisis de dureza y metalográfico.

A continuación se muestran los valores de dureza de los aceros, junto con los valores de porcentaje de austenita retenida, medidos para cada tipo de acero después de los tratamientos T1 y T2 (Figs. 5a y b, respectivamente). Antes del tratamiento, la dureza del acero comercial medida fue menor que el límite inferior del campo de aplicación para la dureza Rockwell C según la norma UNE-EN-ISO-6508 (fijado en $20 \mathrm{HRC}$ ). Este bajo valor provoca que la medida no se refleje en la gráfica. La dureza del acero experimental medida antes de los tratamientos fue de 35 HRC. Las durezas medidas después de los tratamientos T1 y T2 para el acero comercial fueron de 22 HRC por debajo del valor correspondiente a una microestructura martensítica (Mangonon, 2001). Sin embargo, para el acero experimental el valor de dureza medido después de los tratamientos T1 y T2 fue de $45 \mathrm{HRC}$, lo que mantiene la posibilidad de que en el acero experimental se haya formado microestructura martensítica. En todas las medidas de dureza, el valor de la dispersión no superó el 5\%.

En el acero experimental se observa una diferencia entre la cantidad de austenita retenida después del tratamiento T1 y del tratamiento T2. Sin embargo, también se observa que esta diferencia en el porcentaje de austenita retenida no influye en los valores de dureza del acero experimental obtenidos después de ambos tratamientos.

Para el acero comercial, las micrografías obtenidas no muestran microestructura martensítica ni antes ni después de los tratamientos T1 y T2. El acero comercial, en el estado de recepción, muestra amplias zonas de ferrita (Fig. 6a), indicadas por las flechas sólidas negras. En la micrografía correspondiente al acero comercial después del tratamiento T1 (Fig. 6b), se observan zonas claras junto con fases oscuras y alargadas, que corresponden a ferrita y bainita, respectivamente, sin detectarse fase martensítica. La micrografía obtenida para el tratamiento T2 (Fig. 6c) muestra una microestructura similar a la anterior, en la cual se advierte la presencia de bainita (indicada por flechas punteadas negras) y donde tampoco se observa la presencia de martensita.

La micrografía obtenida para el acero experimental, antes de ser sometido a los tratamientos térmicos (Fig. 7a), no presenta ninguna estructura martensítica, pero sí se observa microestructura característica de la martensita después de ser sometido a los tratamientos T1 y T2 (Figs. 7 b y c, respectivamente). No se aprecian diferencias significativas entre las micrografías de los dos tratamientos, a pesar que el análisis por difracción determinó una mayor presencia de austenita retenida en el acero después del tratamiento T1 que del tratamiento T2.

\subsection{Análisis de la estabilidad dimensional}

En cuanto al análisis de la estabilidad dimensional, se observa que no hay tensiones externas aplicadas a las probetas durante los dos tratamientos térmicos. Esto significa que la distorsión final de las probetas de acero comercial y experimental no tienen origen mecánico, por lo que las componentes $\varepsilon_{i, j}{ }^{\mathrm{P}}$ y $\varepsilon_{\mathrm{i}, \mathrm{j}}{ }^{\mathrm{E}}$ (Ec.1) son despreciables en el valor total de la deformación. De igual forma, la ausencia de tensiones externas durante el periodo de austenización implica que la componente 


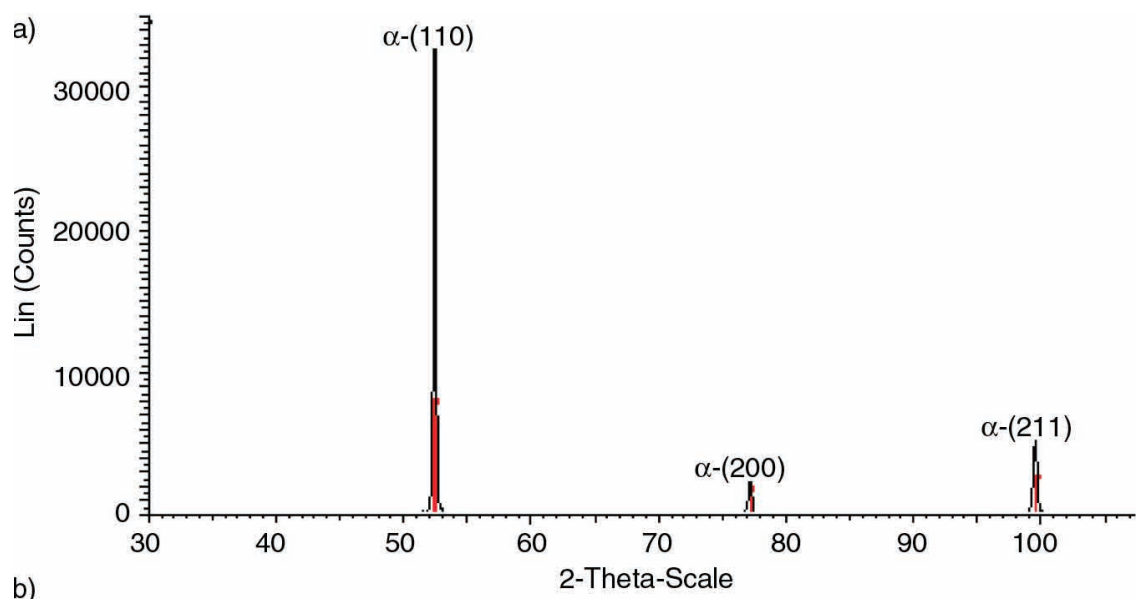

b)
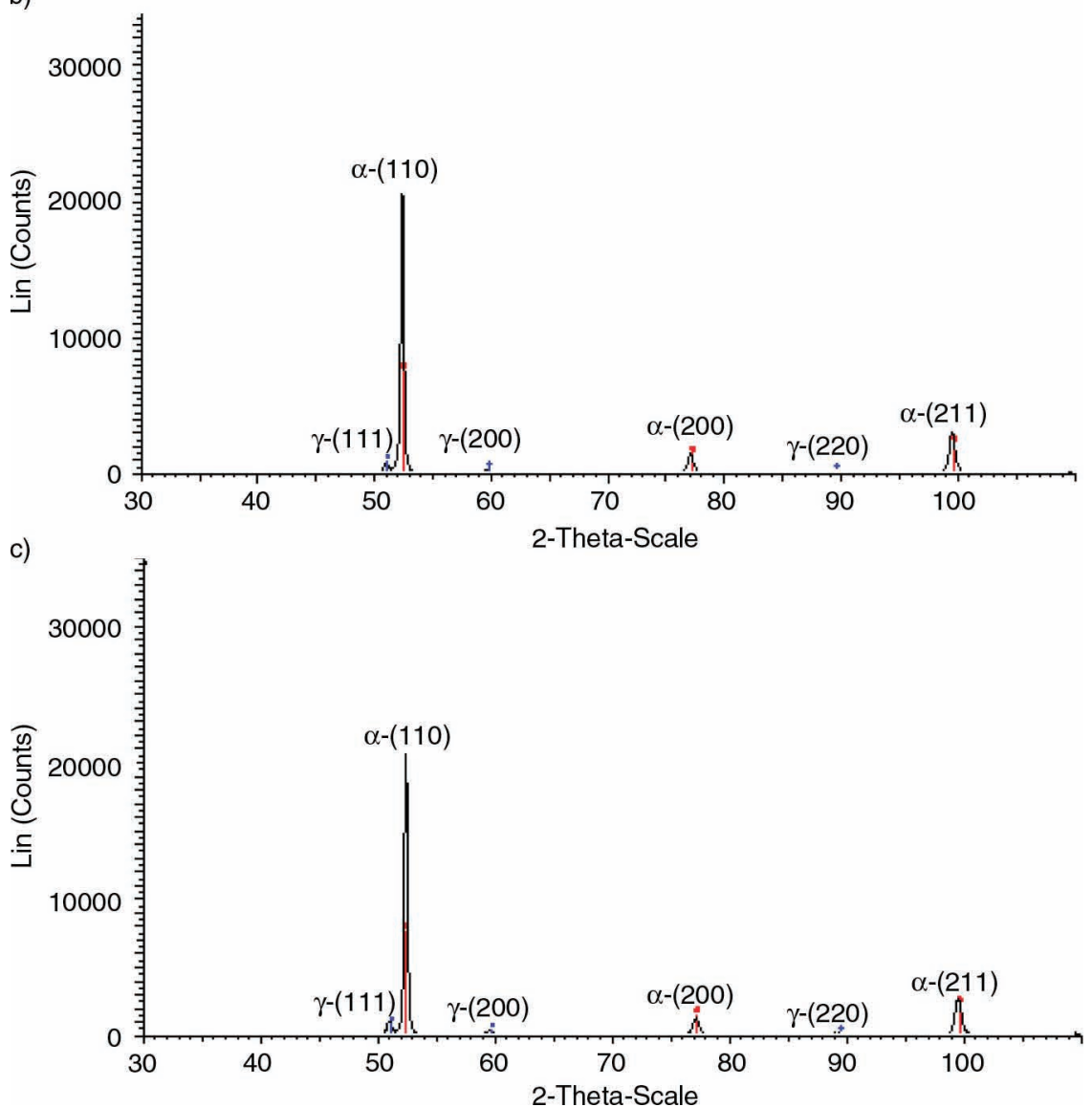

FIGURA 3. Diagramas XRD para el acero comercial: a) en estado de recepción; b) después del tratamiento $\mathrm{T} 1 \mathrm{y}$ c) después del tratamiento T2.

de deformación por fluencia en caliente $\varepsilon_{\mathrm{i}, \mathrm{j}}{ }^{\mathrm{C}}$ también es despreciable. Por tanto, las únicas componentes de deformación asociadas a la distorsión final de las probetas van a ser las producidas por factores térmicos $\varepsilon_{\mathrm{i}, \mathrm{j}}^{\mathrm{TH}}$ y deformación plástica causada por la transformación $\varepsilon_{\mathrm{i}, \mathrm{j}}^{\mathrm{TR}}$. El hecho de que el acero comercial no haya sufrido transformación martensítica va a permitir comparar su estabilidad dimensional con la del acero experimental. En el acero comercial, el factor de distorsión con más peso específico es la deformación producida por efectos térmicos durante el enfriamiento. En el acero experimental hay que tener en cuenta, además, la deformación producida por la transformación austenita-martensita que puede afectar a su estabilidad dimensional. 

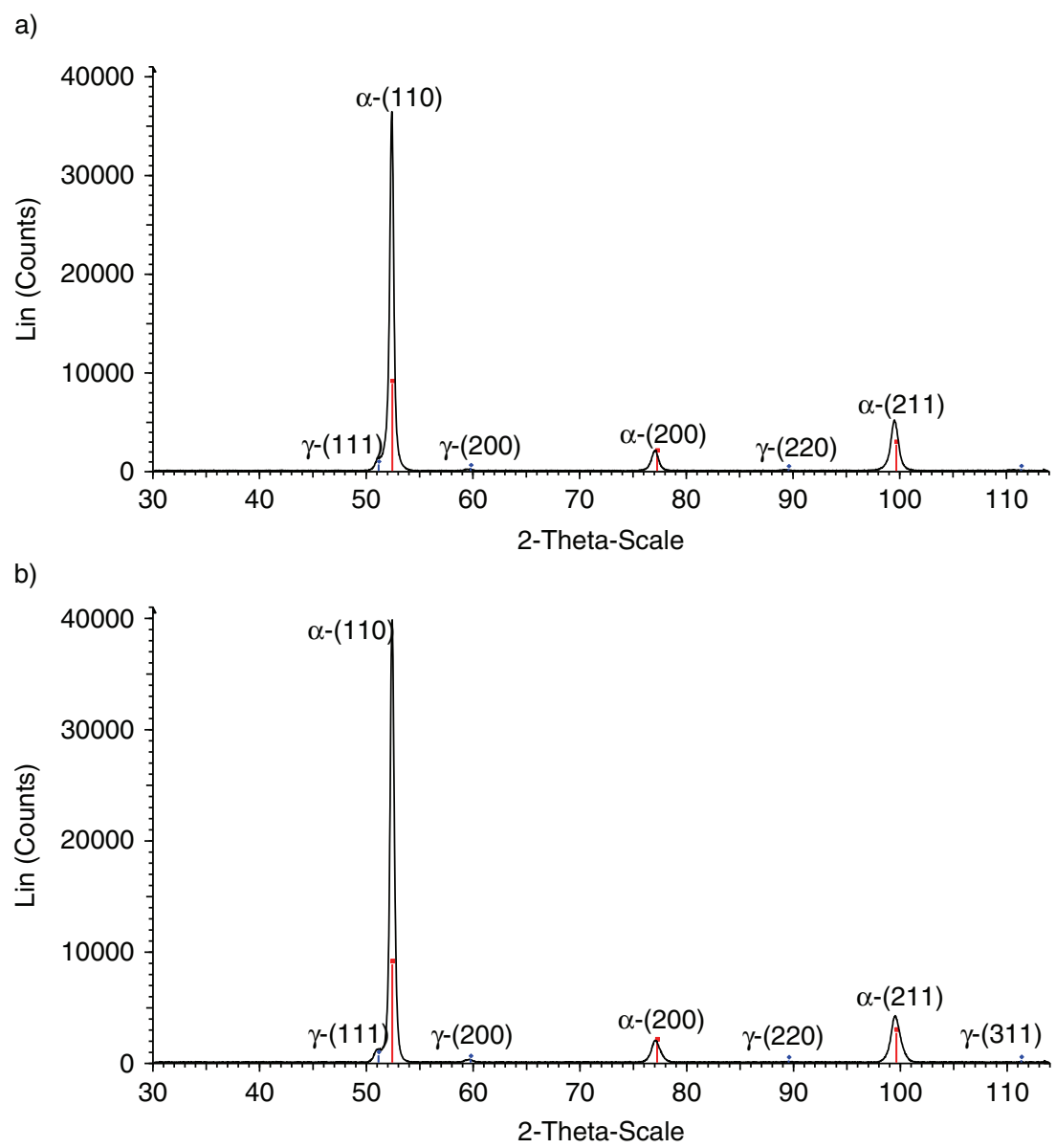

c)

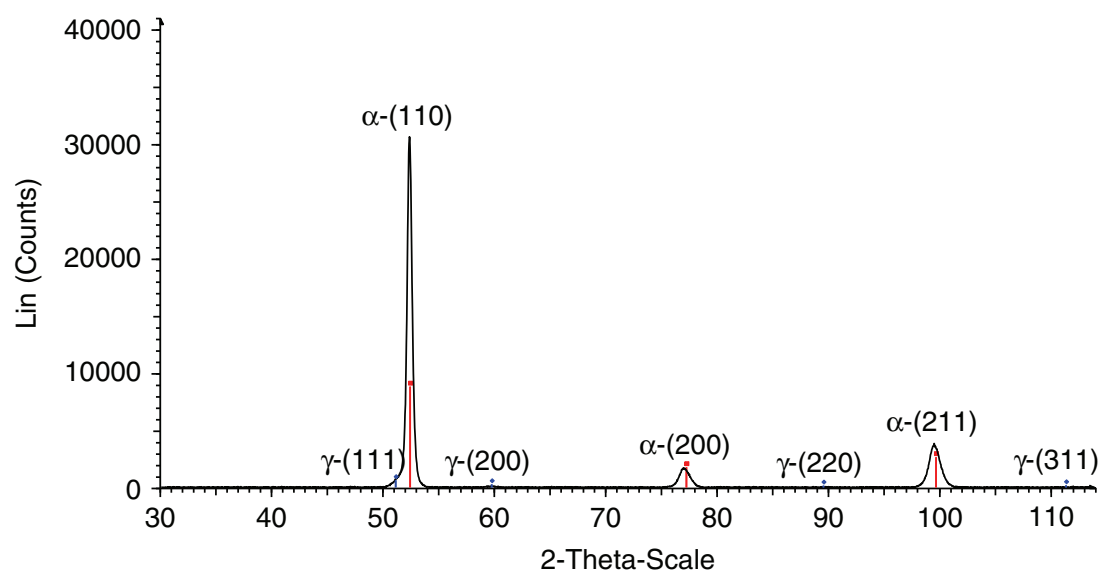

FIGURA 4. Diagramas XRD para el acero experimental: a) en estado de recepción; b) después del tratamiento $\mathrm{T} 1$ y c) después del tratamiento $\mathrm{T} 2$.

a) Cálculo de la variación relativa longitudinal y angular

Una vez realizadas todas las medidas para el estudio de estabilidad dimensional, se obtuvo el valor promedio de la variación relativa lineal y angular para cada acero en cada uno de los tratamientos.
La distorsión de las probetas tiene lugar en el plano perpendicular al eje de la barra. Esto significa que todas las probetas tienen el mismo sentido de corte y mecanizado, no habiendo probetas con direcciones distintas de corte respecto a la barra. En resumen, en la Tabla 2 se muestran los valores promedios de las muestras, obtenidos para cada tipo de acero 
a)

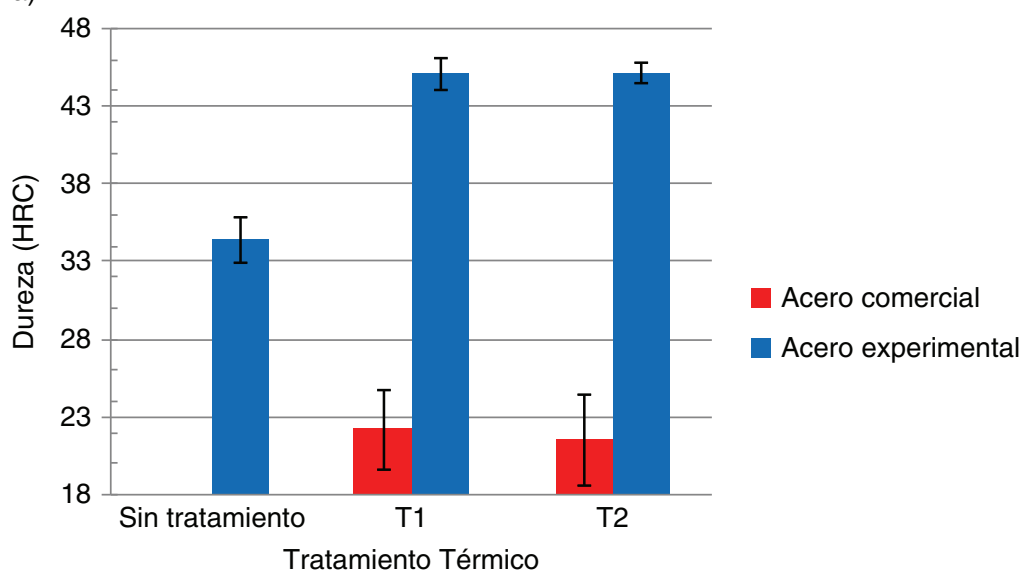

b)

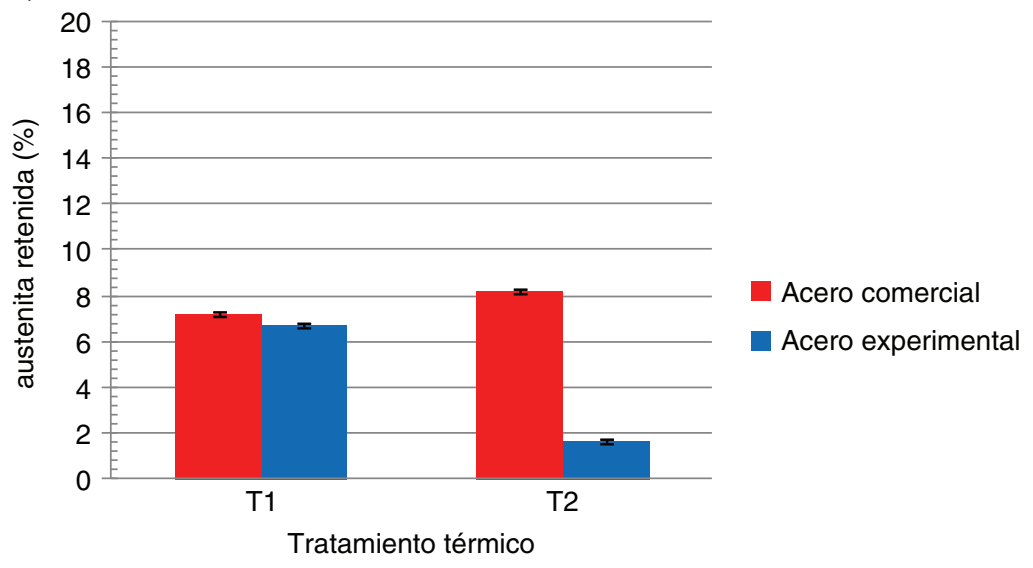

Figura 5. Valores de: a) dureza y b) austenita retenida para los aceros comercial y experimental.

y tratamiento, así como su valor de dispersión, error estándar y, en la última columna, el rango de valores con un nivel de confianza del $95 \%$

Los valores promedios de variación relativa longitudinal (Fig. 8a) para el acero comercial y experimental en el tratamiento T1 fueron $-0,082 \%$ y $0,169 \%$, respectivamente. El valor del acero comercial indica que es más susceptible de sufrir una contracción, al contrario que el acero experimental donde se muestra un valor positivo. Para el tratamiento T2, los valores calculados de distorsión longitudinal relativa fueron $-0,062 \%$ y $0,144 \%$, respectivamente. Los valores muestran que la tendencia se sigue manteniendo, siendo un valor negativo en el caso del acero comercial y positivo en el acero experimental. También se tuvieron en cuenta los valores obtenidos en el cálculo de la distorsión en probetas que no habían sido tratadas previamente (ST). En este caso la distorsión calculada se corresponde con errores implícitos al proceso de medición en las probetas y por ello son despreciables.

También fueron calculadas las variaciones angulares correspondientes a cada acero en cada tratamiento térmico (Fig. 8b). Para el tratamiento T1, los valores promedios obtenidos para el acero comercial y experimental fueron 0,061 y $0,034 \%$, respectivamente. Por otra parte, para el tratamiento T2 los valores calculados fueron 0,055 y $0,002 \%$, respectivamente. Para el acero comercial no hay una diferencia considerable en el valor de la distorsión angular en función del tratamiento térmico, al contrario que ocurre con el acero experimental. Sin embargo, los valores son pequeños y dan a entender que apenas se han producido, en ambos aceros y tratamientos térmicos, distorsiones angulares considerables.

Según se observa en los análisis de los resultados obtenidos, los valores no superan, en valor absoluto, el 0,22 \%. La estabilidad de ambos aceros es buena y las distorsiones de origen térmico y microestructural no son significativas para los enfriamientos realizados en atmósfera forzada de nitrógeno. En un principio se muestra que la distorsión longitudinal ha sido más severa que la angular y, en concreto, los valores de variación obtenidos para el acero experimental han sido mayores que los del acero comercial. Si se comparan los datos obtenidos para la distorsión 

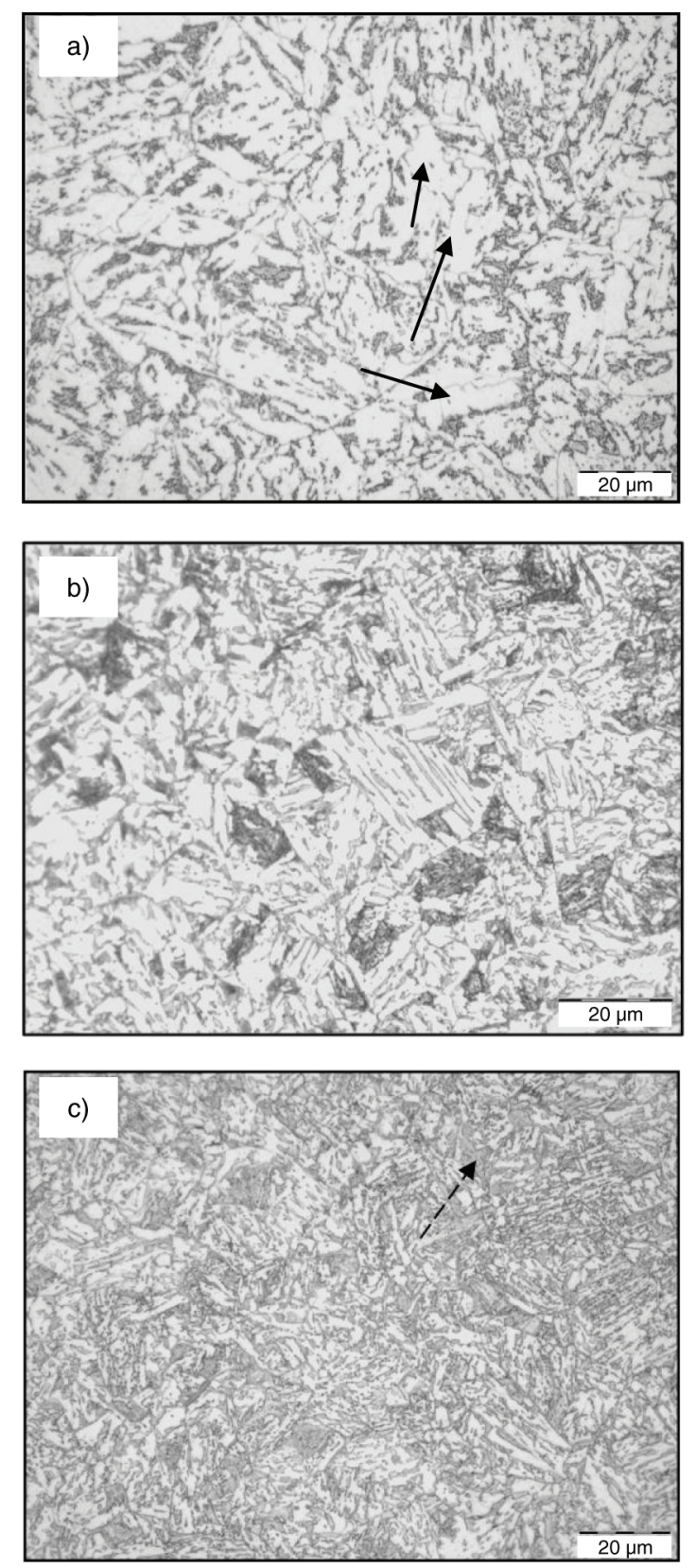

Figura 6. Micrografías obtenidas para el acero comercial: a) en el estado de recepción, donde la flecha sólida negra indica la presencia de ferrita; b) después del tratamiento T1 y c) después del tratamiento T2, donde la flecha punteada indica la presencia de bainita.

longitudinal, se observa que las variaciones calculadas son negativas para el acero comercial, y positivas para el acero experimental. El valor negativo en el acero comercial significa que el factor de distorsión más importante ha sido la contracción debido al enfriamiento durante el proceso térmico. El valor positivo en el acero experimental indica que el origen de la mayor distorsión se debe a la dilatación volumétrica debida a la transformación
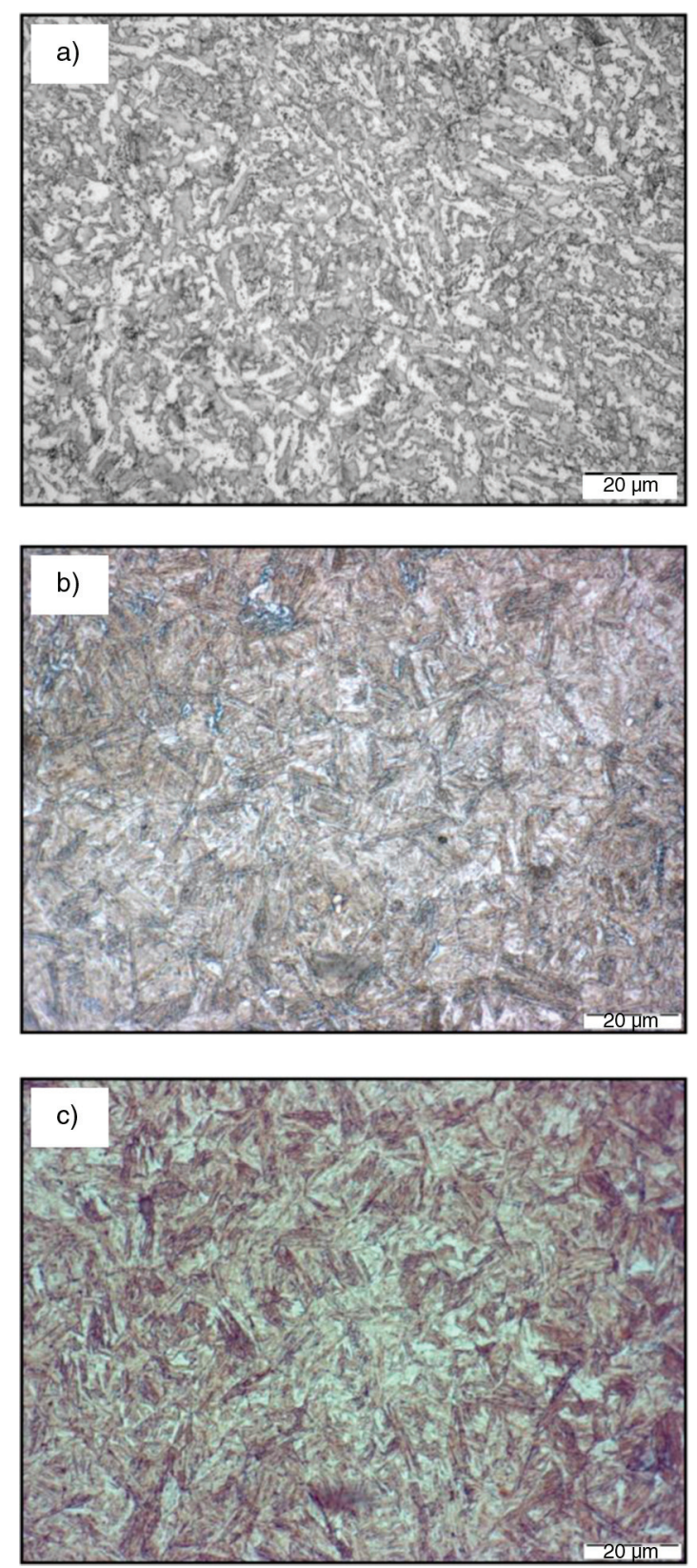

FIgURA 7. Micrografías obtenidas para el acero experimental: a) en el estado de recepción; b) después del tratamiento T1 y c) después del tratamiento $\mathrm{T} 2$.

austenita-martensita, ya que los resultados obtenidos muestran que después de los tratamientos térmicos T1 y T2 el acero experimental ha sufrido una transformación austenita-martensita, mientras que en el acero comercial no se apreciaba presencia de martensita. En resumen, todos los valores absolutos de deformación longitudinal y angular del acero comercial y del experimental están por debajo del $0,22 \%$, lo que demuestra la distorsión total (longitudinal y angular) producida en ambos aceros no es considerable. 
TABLA 2. Datos estadísticos obtenidos de la variación relativa longitudinal y angular de los aceros comercial y experimental en el estado de recepción (ST), tras el tratamiento T1 y tras el tratamiento T2

\begin{tabular}{|c|c|c|c|c|c|c|}
\hline Deformación $^{a}$ & Acero $^{b}$ & Tratamiento & $\begin{array}{c}\text { Variación relativa } \\
\text { media }(\%)\end{array}$ & $\begin{array}{c}\text { Desviación } \\
\text { estándar (\%) }\end{array}$ & Error estándar (\%) & Rango nivel confianza ${ }^{a}$ \\
\hline $\mathrm{L}$ & $\mathrm{C}$ & $\mathrm{ST}$ & $-0,004$ & 0,027 & 0,005 & $(-0,015,0,007)$ \\
\hline $\mathrm{L}$ & $\mathrm{C}$ & $\mathrm{T} 1$ & $-0,082$ & 0,025 & 0,009 & $(-0,099,-0,065)$ \\
\hline $\mathrm{L}$ & $\mathrm{C}$ & $\mathrm{T} 2$ & $-0,062$ & 0,060 & 0,019 & $(-0,099,-0,025)$ \\
\hline $\mathrm{L}$ & $\mathrm{E}$ & ST & 0,005 & 0,025 & 0,006 & $(-0,006,0,016)$ \\
\hline $\mathrm{L}$ & E & $\mathrm{T} 1$ & 0,169 & 0,075 & 0,022 & $(0,127,0,211)$ \\
\hline $\mathrm{L}$ & E & $\mathrm{T} 2$ & 0,144 & 0,078 & 0,026 & $(0,093,0,195)$ \\
\hline A & $\mathrm{C}$ & ST & 0,000 & 0,028 & 0,007 & $(-0,015,0,014)$ \\
\hline A & $\mathrm{C}$ & $\mathrm{T} 1$ & 0,061 & 0,043 & 0,017 & $(0,027,0,095)$ \\
\hline A & $\mathrm{C}$ & $\mathrm{T} 2$ & 0,055 & 0,077 & 0,029 & $(-0,002,0,112)$ \\
\hline A & E & ST & 0,017 & 0,048 & 0,013 & $(-0,008,0,042)$ \\
\hline A & $\mathrm{E}$ & $\mathrm{T} 1$ & 0,034 & 0,103 & 0,037 & $(-0,038,0,105)$ \\
\hline A & E & $\mathrm{T} 2$ & 0,002 & 0,118 & 0,048 & $(-0,092,0,096)$ \\
\hline
\end{tabular}

L: Deformación longitudinal; A: Deformación angular.

C: Acero comercial; E: Acero experimental.

${ }^{\mathrm{a}}$ Nivel confianza $=95 \%$.
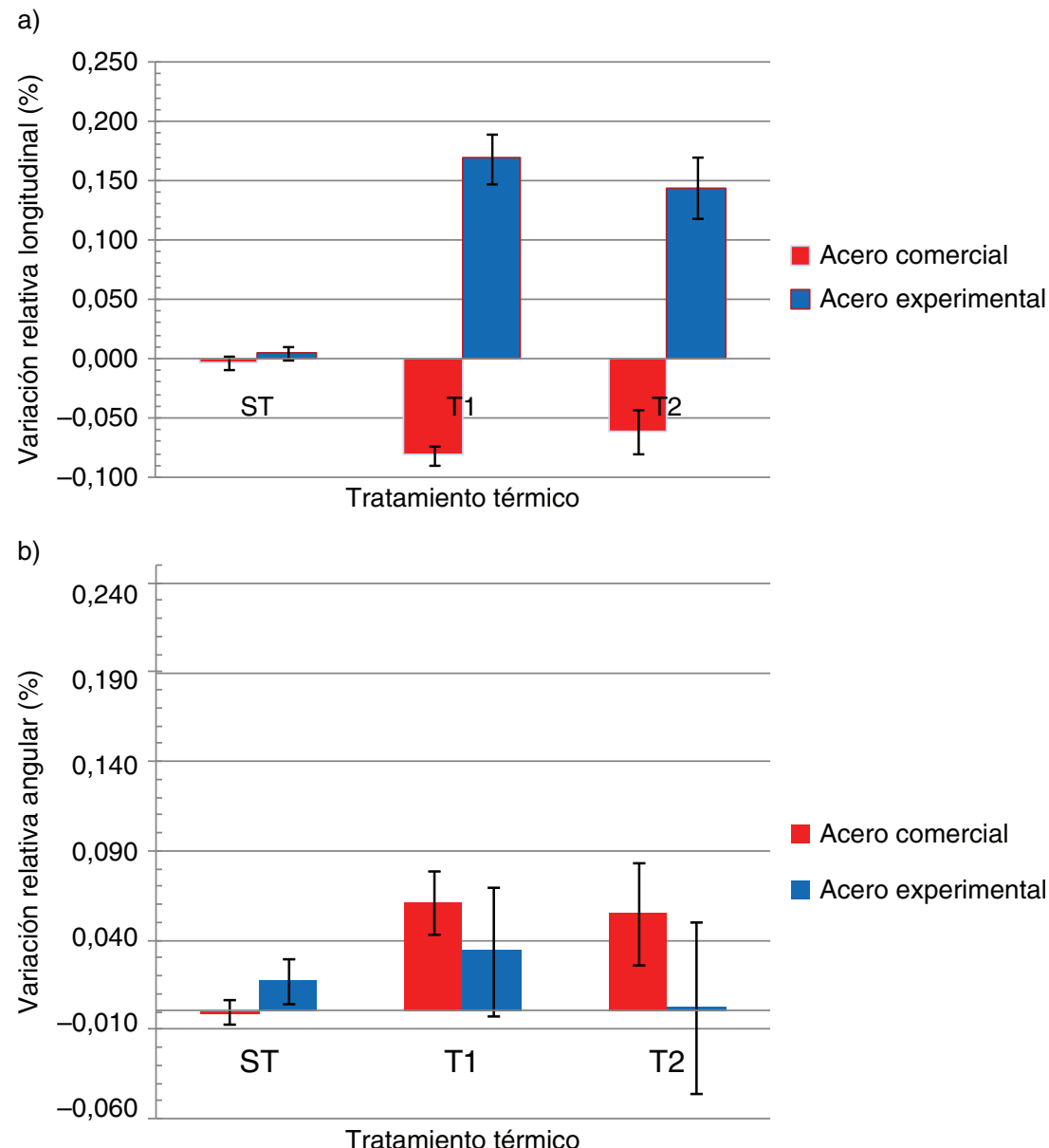

FIGURA 8. Comparación de las variaciones relativas de los aceros comercial y experimental, obtenidas antes y después de los tratamientos térmicos T1 y T2: a) longitudinal y b) angular. 


\section{b) Contraste de hipótesis de los valores obtenidos}

Para comprobar si las diferencias en el valor de las deformaciones obtenidas para el acero comercial y el acero experimental son significativas, se realizó un contraste de hipótesis aplicando estadística no paramétrica, mediante la aplicación de la prueba de suma de rangos de Wilcoxon. A continuación se muestran las hipótesis nulas y alternativas establecidas para los casos estudiados.

\section{Contraste de hipótesis 1:}

- $\mathrm{H}_{01}{ }^{\mathrm{L}}$ : las variaciones longitudinales relativas en los aceros comercial y experimental son idénticas tras ser sometidos ambos al tratamiento T1.

- $\mathrm{H}_{\mathrm{A} 1}^{\mathrm{L}}$ : la variación longitudinal relativa del acero comercial está desplazada a la izquierda o a la derecha de la variación longitudinal relativa del acero experimental tras ser sometidos ambos aceros al tratamiento $\mathrm{T} 1$.

\section{Contraste de hipótesis 2:}

- $\mathrm{H}_{02}{ }^{\mathrm{L}}$ : las variaciones longitudinales relativas en los aceros comercial y experimental, son idénticas tras ser sometidos ambos al tratamiento T2.

- $\mathrm{H}_{\mathrm{A} 2}^{\mathrm{L}}$ : la variación longitudinal relativa del acero comercial está desplazada a la izquierda o a la derecha de la variación longitudinal relativa del acero experimental tras ser sometidos ambos aceros al tratamiento $\mathrm{T} 2$.

Contraste de hipótesis 3:

- $\mathrm{H}_{03}{ }^{\mathrm{L}}$ : las variaciones longitudinales relativas obtenidas en el acero comercial después de los tratamientos T1 y T2 son idénticas.

- $\quad \mathrm{H}_{\mathrm{A} 3}^{\mathrm{L}}$ : la variación longitudinal relativa del acero comercial obtenida después del tratamiento T1 está desplazada a la izquierda o a la derecha de la variación longitudinal relativa correspondiente al tratamiento $\mathrm{T} 2$.

\section{Contraste de hipótesis 4 :}

- $\mathrm{H}_{04}{ }^{\mathrm{L}}$ : las variaciones longitudinales relativas obtenidas en el acero experimental después de los tratamientos $\mathrm{T} 1$ y T2 son idénticas.

- $\mathrm{H}_{\mathrm{A} 4}^{\mathrm{L}}$ : la variación longitudinal relativa del acero experimental obtenida después del tratamiento T1 está desplazada a la izquierda o a la derecha de la variación longitudinal relativa correspondiente al tratamiento $\mathrm{T} 2$.

Aplicando el método, se muestran los resultados obtenidos (Tabla 3) para cada hipótesis nula para confirmar o no lo observado en los estudios de dureza, difracción y metalográficos. Las correspondientes pruebas de contraste rechazan las hipótesis $\mathrm{H}_{01}{ }^{\mathrm{L}} \mathrm{y}$ $\mathrm{H}_{02}{ }^{\mathrm{L}}$, lo que significa que se admiten las hipótesis alternativas, por lo que la diferencia en el tipo de acero sí es significativa en la deformación obtenida después de los tratamientos térmicos T1 y T2. Anteriormente se comprobó que no se había percibido transformación martensítica en el acero comercial, mientras que los valores de dureza y la micrografía revelaban la existencia de martensita en el acero experimental. Se confirma que la transformación austenita-martensita está asociada a una dilatación de volumen proporcional a la fase transformada, por lo que el valor promedio de la deformación longitudinal relativa para el acero experimental es positivo, demostrando así que la transformación microestructural austenitamartensita es el factor más importante de distorsión. Sin embargo, en el acero comercial, la contracción debida al enfriamiento es el principal foco de distorsión, obteniéndose un valor neto negativo.

En las hipótesis $\mathrm{H}_{03}{ }^{\mathrm{L}}$ y $\mathrm{H}_{04}{ }^{\mathrm{L}}$, las pruebas de contraste no rechazan la hipótesis nula. La diferencia en la temperatura de austenización no es significativa en el valor de la deformación relativa longitudinal tanto para el acero comercial como para el acero experimental. En el acero experimental, la cantidad detectada de austenita retenida era menor después del tratamiento T2 $\left(900{ }^{\circ} \mathrm{C}\right.$ temperatura de austenización) que del tratamiento $\mathrm{T} 1\left(860^{\circ} \mathrm{C}\right)$, pero esta diferencia de austenita retenida no influye en el valor final de la deformación. Aun así, los valores absolutos de la deformación del acero comercial y del acero experimental están por debajo del $0,22 \%$, lo que demuestra que la distorsión longitudinal producida en el acero experimental durante la transformación no ha sido lo suficientemente severa.

De igual forma, para analizar la dependencia en el material y en el tratamiento para la variación angular, se volvió a plantear la siguiente serie de hipótesis nulas.

\section{Contraste de hipótesis 5:}

- $\mathrm{H}_{05}{ }^{\mathrm{A}}$ : las variaciones angulares relativas de los aceros comercial y experimental son idénticas tras ser sometidos ambos al tratamiento $\mathrm{T} 1$.

- $\mathrm{H}_{\mathrm{A} 5} \mathrm{~A}$ : la variación angular relativa del acero comercial está desplazada a la izquierda o a la derecha de la variaciones angular relativa del acero experimental tras ser sometidos ambos aceros al tratamiento $\mathrm{T} 1$.

Contraste de hipótesis 6:

- $\mathrm{H}_{06}{ }^{\mathrm{A}}$ : las variaciones angulares relativas de los aceros comercial y experimental, son idénticas tras ser sometidos ambos al tratamiento T2.

- $\mathrm{H}_{\mathrm{A} 6} \mathrm{~A}$ : la variación longitudinal relativa del acero comercial está desplazada a la izquierda o a la derecha de la variación longitudinal relativa 
TABLA 3. Datos obtenidos del contraste de hipótesis para las deformaciones longitudinales

\begin{tabular}{|c|c|c|c|c|c|c|c|c|}
\hline \multirow{2}{*}{$\begin{array}{l}\text { Contraste } \\
\text { Aceros }^{\mathrm{a}} \\
\end{array}$} & \multicolumn{2}{|c|}{1} & \multicolumn{2}{|c|}{2} & \multicolumn{2}{|c|}{3} & \multicolumn{2}{|c|}{4} \\
\hline & $\mathbf{C}$ & $\mathbf{E}$ & $\mathrm{C}$ & $\mathbf{E}$ & $\mathbf{C}$ & $\mathbf{C}$ & $\mathbf{E}$ & $\mathbf{E}$ \\
\hline Tratamiento térmico & T1 & T1 & T2 & T2 & T1 & T2 & T1 & T2 \\
\hline Hipótesis nula & \multicolumn{2}{|c|}{$\mathrm{H}_{01}{ }^{\mathrm{L}}$} & \multicolumn{2}{|c|}{$\mathrm{H}_{02}{ }^{\mathrm{L}}$} & \multicolumn{2}{|c|}{$\mathrm{H}_{03}{ }^{\mathrm{L}}$} & \multicolumn{2}{|c|}{$\mathrm{H}_{04}^{\mathrm{L}}$} \\
\hline Hipótesis alternativa & \multicolumn{2}{|c|}{$\mathrm{H}_{\mathrm{A} 1}^{\mathrm{L}}$} & \multicolumn{2}{|c|}{$\mathrm{H}_{\mathrm{A} 2}^{\mathrm{L}}$} & \multicolumn{2}{|c|}{$\mathrm{H}_{\mathrm{A} 3}{ }^{\mathrm{L}}$} & \multicolumn{2}{|c|}{$\mathrm{H}_{\mathrm{A} 4}^{\mathrm{L}}$} \\
\hline Valor estadístico $S_{1,2}$ & \multicolumn{2}{|c|}{36} & \multicolumn{2}{|c|}{134} & \multicolumn{2}{|c|}{36} & \multicolumn{2}{|c|}{88} \\
\hline Estadístico crítico seleccionado $\left(\mathrm{S}_{\mathrm{L}}\right.$ o $\left.\mathrm{S}_{\mathrm{U}}\right)$ & \multicolumn{2}{|c|}{$\mathrm{S}_{\mathrm{L}}=54$} & \multicolumn{2}{|c|}{$\mathrm{S}_{\mathrm{U}}=114$} & \multicolumn{2}{|c|}{$\mathrm{S}_{\mathrm{U}}=98$} & \multicolumn{2}{|c|}{$\mathrm{S}_{\mathrm{U}}=114$} \\
\hline Comparación & \multicolumn{2}{|c|}{$\mathrm{S}_{1,2} \leq \mathrm{SL}$} & \multicolumn{2}{|c|}{$\mathrm{S}_{1,2} \leq \mathrm{SU}$} & \multicolumn{2}{|c|}{$\mathrm{S}_{1,2} \leq \mathrm{SU}$} & \multicolumn{2}{|c|}{$\mathrm{S}_{1,2} \leq \mathrm{SU}$} \\
\hline Rechazo de la hipótesis nula & \multicolumn{2}{|c|}{ SI } & & & \multicolumn{2}{|c|}{ NO } & & \\
\hline
\end{tabular}

C: Acero comercial; E: Acero experimental.

del acero experimental tras ser sometidos ambos aceros al tratamiento $\mathrm{T} 2$.

Contraste de hipótesis 7:

- $\mathrm{H}_{07}{ }^{\mathrm{A}}$ : las variaciones angulares relativas obtenidas en el acero comercial después de los tratamientos T1 y T2 son idénticas.

- $\mathrm{H}_{\mathrm{A} 7}{ }^{\mathrm{A}}$ : la variación angular relativa del acero comercial obtenida después del tratamiento T1 está desplazada a la izquierda o a la derecha de la variación angular relativa correspondiente al tratamiento T2.

Contraste de hipótesis 8:

- $\mathrm{H}_{08}{ }^{\mathrm{A}}$ : las variaciones angulares relativas obtenidas en el acero experimental después de los tratamientos T1 y T2 son idénticas.

- $\mathrm{H}_{\mathrm{A} 8}{ }^{\mathrm{A}}$ : la variación angular relativa del acero experimental obtenida después del tratamiento T1 está desplazada a la izquierda o a la derecha de la variación angular relativa correspondiente al tratamiento $\mathrm{T} 2$.

En la Tabla 4 se muestra que la hipótesis nula no se rechaza para ninguno de los cuatro casos. La deformación o distorsión angular obtenida no es significativa ni para el tipo de acero enfriado ni para el tratamiento utilizado. La suavidad del enfriamiento, unido a la ausencia de tensiones externas, produce que las distorsiones apreciables sean únicamente de tipo longitudinal, relacionadas con expansiones o compresiones por factores térmicos y por la transformación microestructural, respectivamente.

En definitiva, se puede afirmar que el tipo de acero sí es un factor sensible a la distorsión longitudinal, siendo la transformación austenitamartensita el factor de distorsión más importante en el acero experimental. Por otro lado, el tipo de tratamiento térmico aplicado (T1 o T2) no es un factor influyente en la estabilidad dimensional del acero experimental, y la distorsión angular es despreciable para cada tipo de acero y tratamiento.

Por otra parte, los contrastes de hipótesis realizados muestran que sólo se pueden rechazar las hipótesis nulas correspondientes a la igualdad de variación longitudinal entre el acero comercial y longitudinal en el tratamiento T1 y T2. En resumen, a pesar de que el acero experimental haya sufrido mayor transformación microestructural de tipo austenita-martensita respecto del acero comercial, esta transformación no ha originado una diferencia considerable ni en la distorsión longitudinal ni en la angular. Por lo tanto, las distorsiones dimensionales originadas por la velocidad de enfriamiento (contracción) y la transformación microestructural (dilatación) no alcanzan un nivel de deformación considerable después de los tratamientos térmicos.

Por todo esto, y según lo observado en las mediciones de dureza y en los contrastes de hipótesis realizados para el acero experimental, los valores de dureza y estabilidad dimensional longitudinal registrados son similares en ambos tratamientos térmicos, lo que significa que la mayor solubilidad de vanadio y niobio a $900^{\circ} \mathrm{C}$ que a $860^{\circ} \mathrm{C}$ no influye ni en la dureza ni en la estabilidad dimensional longitudinal del acero experimental. Sí hay que añadir, por último, que el nivel de dureza obtenido después de los tratamientos térmicos, en torno a $45 \mathrm{HRC}$, muestran que el acero experimental ha adquirido unos valores de dureza lo suficientemente altos tras ser sometido a enfriamientos suaves durante los tratamientos $\mathrm{T} 1$ y $\mathrm{T} 2$ sin haberse producido una distorsión significativa. Esto es consecuencia del endurecimiento provocado por los carburos de niobio y vanadio precipitados durante las etapas de austenización y posterior enfriamiento.

\section{CONCLUSIONES}

El acero experimental tiene unos valores promedio de temperaturas $\mathrm{Ac}_{1}$ y $\mathrm{Ac}_{3}$ de $726{ }^{\circ} \mathrm{C}$ y $775{ }^{\circ} \mathrm{C}$, respectivamente. Los tratamientos $\mathrm{T} 1$ (temperatura de austenización de $860^{\circ} \mathrm{C}$ ) y $\mathrm{T} 2$ 
TABLA 4. Datos obtenidos del contraste de hipótesis para las deformaciones angulares

\begin{tabular}{|c|c|c|c|c|c|c|c|c|}
\hline \multirow{2}{*}{$\begin{array}{l}\text { Contraste } \\
\text { Aceros }^{\mathrm{a}} \\
\end{array}$} & \multicolumn{2}{|c|}{5} & \multicolumn{2}{|c|}{6} & \multicolumn{2}{|c|}{7} & \multicolumn{2}{|c|}{8} \\
\hline & $\mathbf{C}$ & $\mathbf{E}$ & $\mathbf{C}$ & $\mathbf{E}$ & $\mathbf{C}$ & $\mathbf{C}$ & $\mathbf{E}$ & $\mathbf{E}$ \\
\hline Tratamiento térmico & T1 & T1 & T2 & T2 & T1 & T2 & T1 & T2 \\
\hline Hipótesis nula & \multicolumn{2}{|c|}{$\mathrm{H}_{05}{ }^{\mathrm{A}}$} & \multicolumn{2}{|c|}{$\mathrm{H}_{06}{ }^{\mathrm{A}}$} & \multicolumn{2}{|c|}{$\mathrm{H}_{07}{ }^{\mathrm{A}}$} & \multicolumn{2}{|c|}{$\mathrm{H}_{08}{ }^{\mathrm{A}}$} \\
\hline Hipótesis alternativa & \multicolumn{2}{|c|}{$\mathrm{H}_{\mathrm{A} 5}{ }^{\mathrm{A}}$} & \multicolumn{2}{|c|}{$\mathrm{H}_{\mathrm{A} 6}{ }^{\mathrm{A}}$} & \multicolumn{2}{|c|}{$\mathrm{H}_{\mathrm{A} 7}^{\mathrm{A}}$} & \multicolumn{2}{|c|}{$\mathrm{H}_{\mathrm{A} 8}{ }^{\mathrm{A}}$} \\
\hline Valor estadístico $S_{1,2}$ & \multicolumn{2}{|c|}{51} & \multicolumn{2}{|c|}{37} & \multicolumn{2}{|c|}{37} & \multicolumn{2}{|c|}{41} \\
\hline Estadístico crítico seleccionado $\left(\mathrm{S}_{\mathrm{L}}\right.$ o $\left.\mathrm{S}_{\mathrm{U}}\right)$ & \multicolumn{2}{|c|}{$S_{U}=61$} & \multicolumn{2}{|c|}{$S_{U}=56$} & \multicolumn{2}{|c|}{$\mathrm{S}_{\mathrm{U}}=56$} & \multicolumn{2}{|c|}{$S_{U}=61$} \\
\hline Comparación & \multicolumn{2}{|c|}{$\mathrm{S}_{1,2} \leq \mathrm{SU}$} & \multicolumn{2}{|c|}{$\mathrm{S}_{1,2} \leq \mathrm{SU}$} & \multicolumn{2}{|c|}{$\mathrm{S}_{1,2} \leq \mathrm{SU}$} & \multicolumn{2}{|c|}{$\mathrm{S}_{1,2} \leq \mathrm{SU}$} \\
\hline Rechazo de la hipótesis nula & \multicolumn{2}{|c|}{ NO } & \multicolumn{2}{|c|}{ NO } & \multicolumn{2}{|c|}{$\mathrm{NO}$} & \multicolumn{2}{|c|}{ NO } \\
\hline
\end{tabular}

C: Acero comercial; E: Acero experimental.

$\left(900{ }^{\circ} \mathrm{C}\right)$ son adecuados para obtener la transformación martensítica.

El valor de la austenita retenida en el acero experimental, después de los tratamientos térmicos T1 y T2, no sobrepasa el $10 \%$, lo que demuestra un buen grado de transformación de la fase austenítica. Las medidas de dureza (45 HRC) y las micrografías revelan que el acero experimental ha sufrido una transformación austenita-martensita. En el acero comercial no se observa microestructura martensítica después de los tratamientos T1 y T2.

Los valores promedios de variación relativa longitudinal para el acero comercial y el experimental en el tratamiento T1 fueron de $-0,082 \%$ y $0,169 \%$, respectivamente. Para el tratamiento T2, los valores calculados de distorsión longitudinal relativa fueron, a su vez, de $-0,062 \%$ y $0,144 \%$, respectivamente La distorsión relativa longitudinal en los aceros comercial y experimental no sobrepasa el $0,20 \%$.

Los valores promedios de variación relativa angular para el tratamiento T1 obtenidos fueron de 0,061 para el acero comercial y $0,034 \%$ para el acero experimental. Por otra parte, para el tratamiento $\mathrm{T} 2$, los valores calculados fueron de 0,055 y $0,002 \%$, respectivamente. La distorsión angular no es considerable para ambos aceros y tratamientos.

La mayor solubilidad de vanadio y niobio a $900{ }^{\circ} \mathrm{C}$ que a $860{ }^{\circ} \mathrm{C}$ no influye en la dureza y a la estabilidad dimensional del acero experimental.

El acero experimental, tras ser sometido a enfriamientos suaves en los tratamientos $\mathrm{T} 1$ y $\mathrm{T} 2$, ha adquirido unos valores de dureza lo suficientemente altos, con una distorsión no significativa, como consecuencia del endurecimiento provocado por los carburos de niobio y vanadio precipitados durante las etapas de austenización y posterior enfriamiento.

\section{AGRADECIMIENTOS}

Este trabajo ha sido subvencionado por el Ministerio de Ciencia e Innovación del Gobierno de España mediante el proyecto PET2007_0475_02.

\section{REFERENCIAS}

Arimoto, K., Yamanaka, S., Narazaki, M., Funatani, K. (2009). Explanation of the origin of quench distortion and residual stress in specimens using computer simulation. Int. J. Microstruct. Mater. Prop. 4 (2), 168-186. http://dx.doi. org/10.1504/IJMMP.2009.028631.

Baker, T.N. (2009). Processes, microstructure and properties of vanadium microalloyed steels. Mater. Sci. Tech. 25 (9), 1083-1107. http://dx.doi.org/10.1179/174328409X453253.

Bhadeshia, H.K.D.H. (2002). Materials Factor Handbook of residual stress and deformation of steel, Ed. ASM International, Materials Park, USA, pp. 3-10.

Canale, L.C., Totten, G.E. (2005). Overview of distortion and residual stress due to quench processing. Part I: factors affecting quench distortion. Int. J. Mater. Prod. Tec. 24 (1-4), 4-52. http://dx.doi.org/10.1504/IJMPT.2005.007941.

Fernández, B.J., Damborenea, J., Ruiz, J. (2002). Effect of hightemperature surface hardening of metallic materials on their dimensional stability. Mater. Design. 23 (4), 377-383. http://dx.doi.org/10.1016/S0261-3069(02)00002-X.

Fossaert, C., Rees, G., Maurickx, T., Bhadeshia, H.K.D.H. (1995). The effect of niobium on the hardenability of microalloyed austenite. Metall. Mater. Trans. A 26 (1), 21-30. http:// dx.doi.org/10.1007/BF02669791.

García-Mateo, C., Sourmail, T., Caballero, F.G., Capdevila, C., García de Andrés, C. (2005). New approach for the bainite start temperature calculation in steels. Mater. Sci. Tech. 21 (8), 934-940. http://dx.doi.org/10.1179/174328405X51622.

Gladman T. (1966). On the Theory of the effect of precipitate particles on grain growth in metals. P. Roy. Soc. Lond. A Mat. Phy. 294, 298-309. http://dx.doi.org/10.1098/ rspa.1966.0208.

Mangonon, P.L. (2001). Procesos térmicos: tratamientos térmicos, Ciencia de Materiales: Selección y Diseño, Ed. Pearson Educación, Méjico D.F, México, pp. 316-388.

Mendenhall, W., Sincich, T. (1997). Probabilidad y Estadística para Ingeniería y Ciencias, Ed. Prentice Hall, México D.F., México.

Montgomery, D.C., Runger, G.C. (2003). Applied statistics and probability for engineers, Ed. John Wiley and Sons, Nueva York, USA.

Nallathambi, A.K., Kaymak, Y., Specht, E., Bertram, A. (2010). Sensitivity of material properties on distortion and residual stresses during metal quenching processes. J. Mater. Process. Tech. 210 (2), 204-211. http://dx.doi.org/10.1016/j. jmatprotec.2009.09.001.

Norma UNE-EN-ISO-6508 (2007). Aceros Metálicos: ensayos de dureza Rockwell, Ed. AENOR, Madrid, España.

Paquin, R.A. (1990). Dimensional Stability: an overview, Proceedings of. SPIE, Society of Photo-optical Instrumentation Engineers, Vol. 1335, Bellingham, USA, pp. 2-19.

Qiu, H., Qi, J., Yin, F., Hiraoka, K. (2009). Determination of parameters for fitting the dilatation curve of austenitemartensite transformation in $\mathrm{Cr}-\mathrm{Ni}$ steels. ISIJ Int. 49 (1), 146-148. http://dx.doi.org/10.2355/isijinternational.49.146. 
Rees, G.I., Perdrix, J., Maurickx, T., Bhadeshia, H.K.D.H. (1995). The effect of niobium in solid solution on the transformation kinetics of bainite. Mat. Sci. Eng. A-Struct. 194 (2), 179-186. http://dx.doi.org/10.1016/0921-5093(94) 09673-2.

Rietveld, H.M. (1969). A profile refinement method for nuclear and magnetic structures. J. Appl. Crystallogr. 2 (2), 65-71. http://dx.doi.org/10.1107/S0021889869006558.

San Martín, D. Caballero, F.G., Capdevila, C. García-deAndrés, C. (2006). Estudio y modelización de la influencia de las partículas de segunda fase sobre el crecimiento de grano austenítico en un acero microaleado con niobio. Rev. Metal. 42 (2), 128-137. http://dx.doi.org/10.3989/revmetalm.2006.v42.i2.13

Santofimia, M.J. (2006). La transformación bainítica sin formación de carburos en aceros, Tesis Doctoral, Departamento de Física de Materiales, Universidad Complutense (UCM), Madrid, España.
Skoog, D.A., Crouch, S.R., Holler, F.J. (2007). Principles of Instrumental Analysis, Ed. Thomson-Brooks/Cole, Belmont, USA.

Steven, W., Haynes, A. G. (1956). The temperature of formation of martensite and bainite in low alloy steels. J. Iron Steel Inst. 183, 349-359.

Telher M. (2009). Modeling phase transformations and volume changes during cooling of case hardening steels, Tesis de Licenciatura, Department of Materials Science and Engineering, Royal Institute of Technology (KTH), Estocolmo, Suecia.

Yang, H.S, Bhadeshia, H.K.D.H. (2009). Austenite grain size and the martensite-start temperature. Scripta Mater. 60 (7), 493 495. http://dx.doi.org/10.1016/j.scriptamat.2008.11.043.

Yao, X., Zhu, L., Li, V. (2004). Finite element analysis of residual stress and distortion in an eccentric ring induced by quenching, Proceedings of the $14^{\text {th }}$ IFHTSE Congress, Vol. 25, Transactions of Materials and Heat Treatment, Beijing, China, pp. 746-751. 